\title{
Error Resilient SPIHT Coded Grayscale Image transmission over MIMO System
}

\author{
Rohit Kumar Murarka ${ }^{1}$, Anurag Mishra ${ }^{2}$ and Kanchan Sharma ${ }^{3}$ \\ ${ }^{1}$ Department of Electronics and Communication Engineering, IGIT, GGSIPU, \\ New Delhi, India \\ ${ }^{2}$ Department of Electronics, Deendayal Upadhyay College, University of Delhi, \\ New Delhi, India \\ ${ }^{3}$ Department of Electronics and Communication Engineering, IGDTUW, New \\ Delhi, India \\ ${ }^{1}$ rohitkumarmurarka@gmail.com,2anurag_cse2003@yahoo.com \\ ${ }^{3}$ sharma_kanchan@yahoo.com

\begin{abstract}
In this paper, we propose Error Resilient SPIHT coded MIMO system to address the issue of image transmission and reception over noisy Rayleigh Fading channel. Set Partitioning in Hierarchical Trees (SPIHT) is chosen as the compression algorithm since it offers the lossless compression and excellentrate distortion in noiseless environment. SPIHT encoded data is modulated in the manner that it will provide Unequal Error Protection (UEP) to achieve error resilience. In proposed method, SPIHT encoded data is divided into the low priority stream (LP) and the high priority stream (HP) to achieve $U E P$ and as a result it yields high PSNR value. These streams are modulated using $M$ QAM modulation scheme and transmitted over Multiple Input Multiple Output (MIMO) system to achieve better diversity. The adaptlue alue of $M$ is determined by the proposed algorithm. Simulation results depicts that PSNR of received images are better as compared to that reported by Fowdur et al [12].
\end{abstract}

Keywords: SPINT, MIMO, Rayleigh Fading Channel, EEP, UEP, PSNR

\section{Introdaction}

With advances in digita wireless communication, data transfer is not only restricted to text, but it includes audjo and images. Out of these images and videos occupy most of the available channel bandwidth during its transmission over medium in real time scenario. This motivates to develop an efficient algorithm that reduces complexity and bandwidth requiremênts.

Over the years, many image source coders have been introduced which delivers PSNR scalable bit streams in an efficient time bound manner. The encoders achieve better fidelity with fewer bits. But at the same time these source coder outputs are also susceptible to channel noise and even a single bit error can sometimes render the image useless. In general, wireless channels are noisy and transmitted bit stream suffers multiple errors. This makes it important to develop a robust mechanism to protect bit stream from these errors.

There exists a number of image compression techniques. JPEG is quiet popular among them and is a DCT based algorithm. Lossy JPEG compression degrades a lot at high compression ratios, with noticeable artifacts at block boundaries. Also, the quantization error causes objectionable 'ringing' around sharp edges, especially in text.

Said and Pearlman introduced the Discrete Wavelet Transform (DWT) based Set Partitioning in Hierarchical Trees (SPIHT) which is the most well-known EZW derivative 
[1]. SPIHT is known to offer better image quality than JPEG, especially at higher compression ratios. It inherits the lossless compression as well as excellent rate distortion in noiseless environment. We use SPIHT source coder bit stream for introducing error resilience over a wireless channel.

Bit stream can be protected by Unequal Error Protection (UEP) as proposed by Pelz where different parts of encoded stream have unequal importance [2]. Generally speaking, there are two ways to achieve UEP.

First way to achieve UEP is channel coding as cited by Modestino and Daut where different channel coding is employed in different parts of encoded stream based on their importance [3]. Another channel source coding viz., Rate Compatible Punctured Convolutional codes (RCPC) was studied by Hagenauer, where UEP is achieved by puncturing high priority bits less frequently and low priority bits being punctured more frequently [4]. Barbulescu and Pietrobon, used Turbo Codes to achieve UEP in the same manner that of as RCPC [5]. Sherwood and Zeger used progressive image comptession scheme technique by cascading an existing image coder with carefully chosen erfor control coding [6].

The second way to achieve UEP is to rely on different modulation rates as they have different bit error rate susceptibility for same channel SNR 17-8]. Man, Kossentini and Smith [7] introduced the concept of separating the SPIHT coded bit streams into sub streams based on their respective error sensitivity and thereafter the sub streams are processed through three tier UEP. In the three tier UEP scheme, fixed length coding was used for a fixed portion of the SPIHT coded bit stream and the unequal error protection is achieved by employing RCPC channel code on sorting pass (significant map only) and no channel coding is done in the refinement pass. Since, the bit reorganization has been performed on overall bit-streams, thererore it loses its progressiveness property. A theoretical analysis of unequal error protection of SPIHT coded images in presented by Cao [8].

Khan et. al., analysed SPIHT source bit stream characteristics and established that bits can be classified according to their error vulnerabilities [9]. The source bit streams are divided into two sub streams: Critical Bit Stream (CB) and Non Critical Bit Stream (NCB). The error in the $\mathrm{CB}$ stream results in the loss of synchronization and the image may get degraded severely or may not be retrieved at all during decoding at the receiving end. The error in $N C B$ stream cause mild degradation in received image and generally do not disturb the decoding process. Therefore, CB bitstream is a high priority stream and NCB is treated as a low priority stream.

Let us look at similar works done in the past. Fowdur et al., [12] in their work achieved UEP using RCPC with different coding rate for different bit classes as well as prioritized retransmission of important bit classes. Ayyub and Ekram Khan [13] applied UEP using $\mathrm{RCPC}$ with yarying puncturing rate depending on importance of bit streams. Both of these groups cons dered presence of AWGN channel noise in their work. Yap and Ngan [14] applied a mixture of RCPC codes and interleaving to combat the burst errors produced by the fading channel. They considered Rayleigh fading channel noise in the transmission medium.

In this paper, we propose a novel algorithm to transmit the SPIHT coded bit stream using unequal error protection over MIMO system under Rayleigh fading conditions. Here, we separate SPIHT coded bitstream into High Priority stream and Low Priority streams and modulate them with $\mathrm{M}$-ary modulation scheme, where different rate of $\mathrm{M}$ selected for HP and LP stream as per proposed algorithm. Finally it is transmitted over MIMO system and decoded at the receiver. The paper is organised as follows: Sections 2 discusses the basics of SPIHT algorithm. Sections 3 and 4 describe the basics of MIMO and Alamouti OSTBC systems respectively. Section 5 discusses the experimental setup of proposed method followed by results and discussion in section 6 . Section 7 concludes the work. 


\section{Set Partitioning In Hierarchical Trees (SPIHT)}

As the name implies, Set Partitioning In Hierarchical Trees (SPIHT) is based on hierarchical set partitioning method. SPIHT arranges wavelet coefficients as a collection of spatial orientation trees, where each tree consists of coefficients from all sub bands that correspond to the same spatial location in the image. Figure 1 depicts wavelet decomposition and spatial orientation tree. A large number of insignificant coefficients are considered as a set and compactly coded together as an insignificant set symbol instead of coding each insignificant coefficient individually. It also defines a partitioning rule to divide a given significant set into smaller subsets to efficiently extract the significant coefficients.

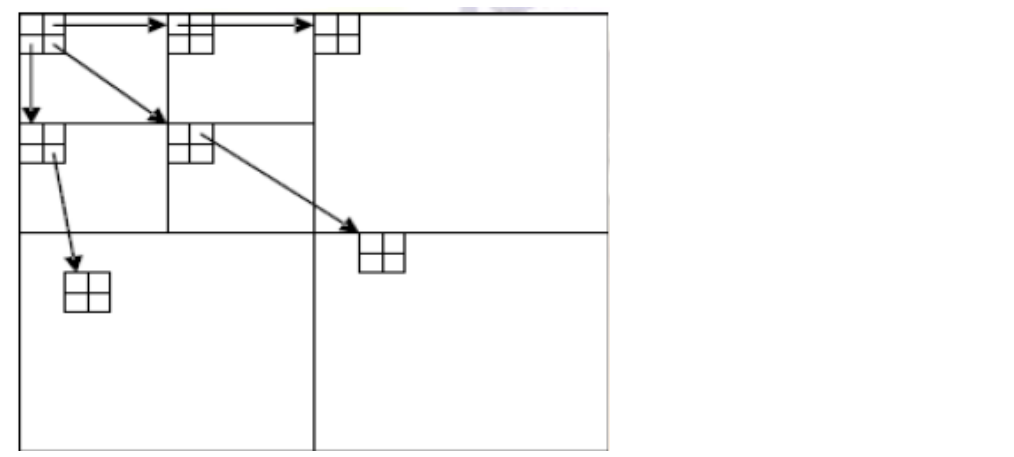

Figure 1. Two-level Wavelet Decompósition and Spatial Orientation Tree (10)

The algorithm works by partitioning the waretet decomposed image into significant partitions based upon the given by Eqn. (1).

$$
S_{n}(\mathbb{C})= \begin{cases}0, & \text { othermise } \\ 1, & \text { if } \max _{(i, j) \in \mathrm{T}}\left\{|Y(i, j)| \geq 2^{n}\right\}\end{cases}
$$

where $T$ is a set of coefficients with values larger than a given threshold. For $S_{n}(T)=$ $0, \mathrm{~T}$ is said to be an insignificant set; else, $\mathrm{T}$ is called significant set. An insignificant set is represented by a single bit " 0 ," whereas a significant set is partitioned into subsets, whose significances are to be tested again.

SPIHT consists of two main stages - sorting pass and refinement pass, executed in the order of appearence. Sorting pass again consists of three linked list elements:

LIP - List of insignificant pixels

LIS - List of insignificant sets

LSP List of significant Pixels

LSP is an empty stream at the start. LIP consists of co-ordinates of all tree roots in the lowest sub band, and LIS keeps all tree roots with nonempty descendent trees which are at the lowest sub band. The first pass i.e., sorting pass scans the LIP and moves all significant coefficients to LSP and stores its sign. Then, it scans LIS executes the significant information following the partitioning sorting algorithms.

The second pass i.e., refining pass scans the coefficients in LSP and produces a single bit output based on the current threshold. Then, threshold is divided by 2 and the encoder executes the two passes again. This procedure is recursively applied until the number of output bits reaches the desired number. The decoding is symmetric and relies upon replacing "output" by "input" only. In a way, it is nearly symmetric to encoder (receives 
largest msb level that encoder finds and outputs). SPIHT bit stream generates an output bit stream with different degree of vulnerability.

Error in non-critical bits degrades the image locally without affecting synchronization between encoder and decoder. On the other hand, errors in critical-bits lead to global error thereby disturbing synchronization between encoder and decoder.

\section{The MIMO System}

The block diagram representation of MIMO transmitter and receiver system is shown in Figure 2. The input stream is passed through coder and is first interleaved. This stream is passed to symbol mapper, say, M-ary QAM modulation and further passed to space time encoder which outputs multiple spatial data stream. Finally, it is transmitted over $\mathrm{N}_{\mathrm{T}}$ antennas. The data is received and mapped over $\mathrm{N}_{\mathrm{R}}$ antennas and is decoded in reverse order w.r.t. the transmitter.

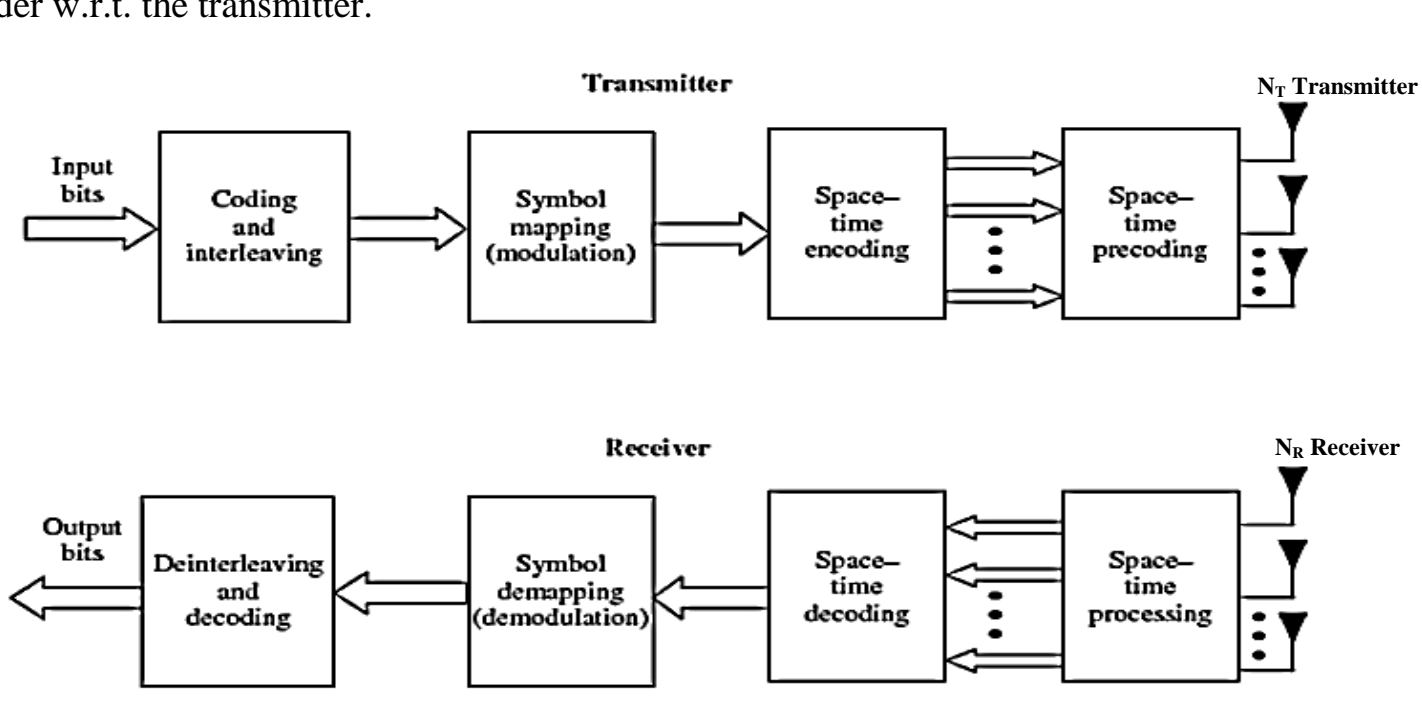

Figure 2. MIMO-Transmitter and Receiver

We assume that number of transmitting antennas is $\mathrm{N}_{\mathrm{T}}$ and $\mathrm{i}^{\text {th }}$ transmitted signal is represented as $(t)$, where i represents the antenna number i.e., $\mathrm{i}=1 \ldots \mathrm{N}_{\mathrm{T}}$. Similarly number of receiyng antennas is $N_{R}$ and $j^{\text {th }}$ received signal is represented as $y_{j}(t)$, where $j$ is represents the antenna number i.e., $\mathrm{j}=1, \ldots \mathrm{N}_{\mathrm{R}}$. The relationship between transmitted and received signal is giyen by Eqn. (2).

$$
\mathrm{y}_{\mathrm{i}}(\mathrm{t})=\sum_{\mathrm{i}=1}^{\mathrm{N}_{\mathrm{T}}} \mathrm{h}_{\mathrm{i}, \mathrm{j}}(\mathrm{t}) * \mathrm{x}_{\mathrm{i}}(\mathrm{t})+\mathrm{n}_{\mathrm{j}}(\mathrm{t}), \quad \mathrm{j}=0,1, \ldots \mathrm{N}_{\mathrm{R}}
$$

where $b_{i, j}(t)$ is the channel impulse response between the $i^{\text {th }}$ transmitter and $j^{\text {th }}$ receiver

The channel impulse response matrix of MIMO is given by Eqn. (3).

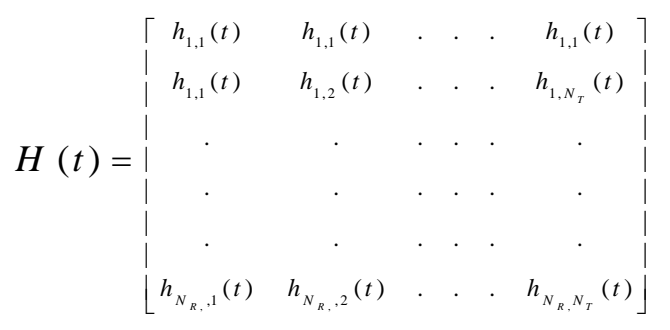


MIMO systems can be used efficiently to achieve transmit or receive diversity depending on whether multiple transmitter are used at transmitter or receiver side. Both could be achieved by using multiple antennas on either side. The MIMO systems thrives on the assumption that there exits an extremely low probability of multiple channels undergoing simultaneous deep fades under various diversity conditions.

\section{Alamouti's Orthogonal Space-Time Block Coding}

The capacity in MIMO system can be exploited further by using spatial diversity, to combat channel fading. This can be achieved by transmitting several replicas of the same information through each antenna. This leads to exponential decrease in the probability of information being lost. The diversity order or diversity gain of a MIMO system is defined as the number of independent receptions of the same signal. A MIMO system with $\mathrm{N}_{T}$ transmit antennas and $\mathrm{N}_{\mathrm{R}}$ receive antennas has potentially full diversity (i.e., maximum. diversity) gain equal to $\mathrm{N}_{\mathrm{T}} \mathrm{N}_{\mathrm{R}}$.

Alamouti published his work on transmit diversity and is the first known Space Time Block Coding method [11]. The Alamouti STBC scheme uses two transmi antennas and $\mathrm{N}_{R}$ receiver antennas and can attain a maximum diversity order $\mathrm{r}_{\diamond}$ of $2 \mathrm{~N}_{\mathrm{k}}$. Also, Alamouti scheme achieves full rate since it transmits symbols every 2 thine intervals. The Alamouti matrix is defined by Eqn. (4).

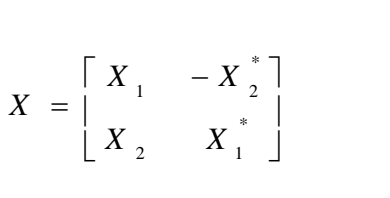

Alamouti scheme belongs to Orthogonalspace-Time Block Coding (OSTBC) class. Orthogonal STBCs are an important subetass of linear STBCs that guarantee that the ML (Minimum Likelihood) detection of different symbols is decoupled and at the same time the transmission scheme achieves diversity onder equal to $\mathrm{N}_{T} \mathrm{~N}_{\mathrm{R}}$.

\section{Our Proposed Method}

The proposed method does not interfere with the encoder process, but efficiently deals with output of SPIHT encoder to deliver relatively good image quality even in the noisy environment.

The SPIAT have three kinked lists LIP, LIS and LSP, already discussed in section 2. On closer inspection, it is revealed that LIP and LIS are Low Priority streams i.e., error in these bits only degrades the image locally. On the other hand, LSP causes global error leading to catastrophic failure of decoding process due to lack of synchronization between encoder and decoder. Further, it is found that position of error in LSP bitstream also matters, i.e. mostly error in first half of stream induces global error while the impact is not severe for the second half. Thus, we assign the first half of the LSP bit stream as a High P(ior)ty stream and the second half as a Low Priority stream. SPIHT header information is needed at SPIHT decoder stage since parameters must be matched to encoder, hence this is also considered as High Priority stream.

Based on the above observations in the proposed algorithm, the SPIHT stream is divided into two sub streams - Low Priority bit stream and High Priority bit stream depending on their error vulnerability. In addition to the usual encoded bitstream, we also take due care of header information which must be matched exactly at receiver side for decoding to be performed. The header contains critical information like image size, type of wavelet decomposition used etc. To preserve header in very noisy condition, we use repeated coding, without any real impact on bandwidth. This is due to the reason that header information is composed of only few bytes.

In line with the UEP scheme, the proposed algorithm modulates Low Priority stream with 16-QAM modulation whereas the High Priority stream is modulated using 4-QAM 
modulation. This modulated data is sent over OSTBC MIMO system through Rayleigh fading channel to achieve better channel diversity as well as spatial diversity. At the decoder, the data is received by NR MIMO receiving antennas. Depending on whether data consists of HP stream or LP stream, it is decoded with 4-QAM or 16-QAM demodulator respectively. The received HP stream and LP stream are passed to the stream combiner which results the stream output in the same fashion as they were before passing into the stream splitter used in the transmitter section. Figure 3 depicts flow chart, exhibiting transmission and receiving process.

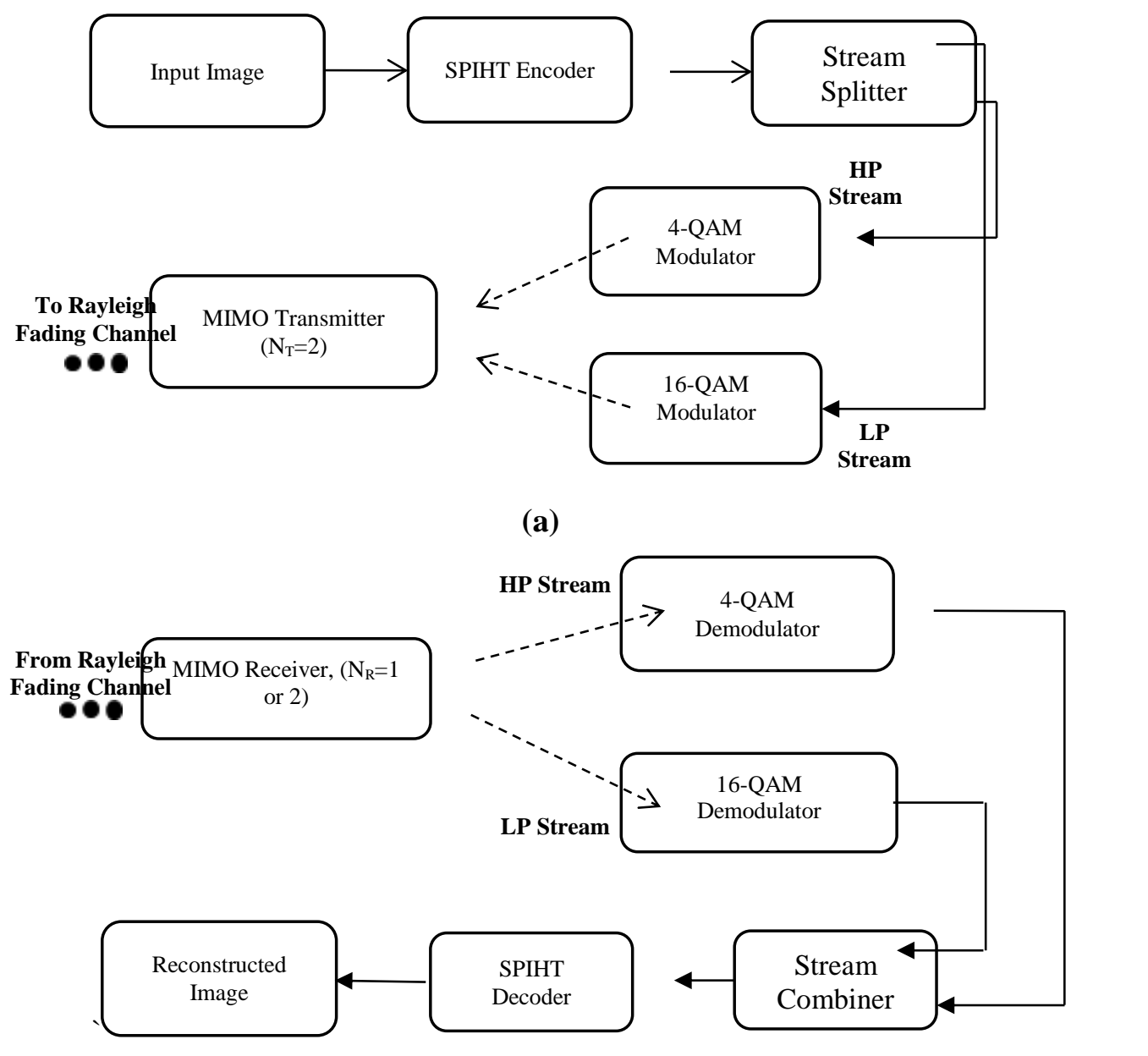

(b)

Figure 3. (a) Encoding and Transmitting the Image over MIMO Rayleigh Faded Channel, (b) Receiving and Decoding to Obtain the Reconstructed Image

We use two 256x256 gray scale images - Lena and Baboon for simulation purpose. The objective is to establish generality and accuracy of results obtained using the proposed method. We first pass the image to SPIHT encoder. The stream is then split into Low Priority Stream and High Priority Stream. The Low Priority and High Priority stream are sent one by one in known order of their respective modulation scheme followed by the MIMO transmitter to the Rayleigh faded channel. At receiver, the transmitted stream is received from Rayleigh channel and arranged into the LP and HP stream in known order w.r.t. transmitter. The streams are then demodulated and combined to form input to the SPIHT decoder. This arranged data stream is further passed to the SPIHT decoder to get back the reconstructed image. To evaluate quality of reconstructed image w.r.t. the 
received image, we calculate PSNR in $\mathrm{dB}$, which is represented by Eqn. (6). The PSNR uses Mean Square Error (MSE) given by Eqn. (5).

$$
\begin{gathered}
M S E=\sum_{i=1}^{x} \sum_{j=1}^{y} \frac{\left(\left|A_{i j}-B_{i j}\right|\right)^{2}}{x * y} \\
P S N R(d B)=10 * \log _{10}\left(\frac{255^{2}}{M S E}\right)
\end{gathered}
$$

where, $A_{\mathrm{ij}}$ represents original image

$B_{i j}$ represents reconstructed image

$\mathrm{x}$ and $\mathrm{y}$ are height and width of the image respectively.

\section{Results and Discussion}

We simulate results for Lena and Baboon using Matlab R2013a. Results for Equal Error Protection (EEP) are compared w.r.t. UEP scheme for 2x1 MIMO and 2 $2 \mathrm{MIMO}$ systems. It is clear that results for UEP for both MIMO systems are better than EEP. Moreover, the results for $2 \times 2$ MIMO systems are observed to be better than 2x1 MIMO systems due to enhanced spatial diversity.

Table 1 compiles the comprehensive simulation results for the two images both for UEP and EEP under MIMO 2x1 and 2x2 configurations. It is verified that by increasing $\mathrm{N}_{\mathrm{R}}$ for MIMO systems, reception quality for images can be further enhanced greatly due to reduction in BER.

Table 1. Reconstructed Image PSNR for bothlena and Baboon 256 bit

\begin{tabular}{|c|c|c|c|c|c|c|c|c|}
\hline \multirow{3}{*}{$\begin{array}{c}\text { Channel } \\
\text { SNR } \\
\text { (in dB) }\end{array}$} & \multicolumn{4}{|c|}{ Lena Image } & \multicolumn{4}{|c|}{ Baboon Image } \\
\hline & \multicolumn{2}{|c|}{$\begin{array}{c}\text { MIMO-2x1 } \\
\left(N_{T}=2, N_{R}=1\right)\end{array}$} & \multicolumn{2}{|c|}{ MIMQ-2 } & \multicolumn{2}{|c|}{$\begin{array}{c}\text { MIMO-2x1 } \\
\left(\mathrm{N}_{\mathrm{T}}=2, \mathrm{~N}_{\mathrm{R}}=1\right)\end{array}$} & \multicolumn{2}{|c|}{$\begin{array}{c}\text { MIMO-2x2 } \\
\left(\mathrm{N}_{\mathrm{T}}=2, \mathrm{~N}_{\mathrm{R}}=2\right)\end{array}$} \\
\hline & EEP & & EEP & UEP & EEP & UEP & EEP & UEP \\
\hline 13 & 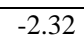 & & & 11.8 & 2.09 & 16.6 & 16.2 & 24.6 \\
\hline 14 & & 18 & 9.5 & 11.8 & 6.3 & 19.5 & 17.2 & 26.4 \\
\hline 15 & 05 & 19. & 14.8 & 30.5 & 9.58 & 21 & 17.8 & 30.3 \\
\hline 16 & 10.1 & & 14.7 & 35.8 & 10.3 & 25.9 & 18.9 & 31.7 \\
\hline 17 & 14 & 2.5 & 25.9 & 33.8 & 17.8 & 27.5 & 21.2 & 31.9 \\
\hline 18 & 13 & 34 & 44 & 44 & 18.5 & 30.5 & 21.5 & 43.3 \\
\hline 19 & 16 & 36.1 & 44.1 & 44.1 & 18.8 & 32 & 43.3 & 43.3 \\
\hline 20 & 21.9 & 34.3 & 44.1 & 44.1 & 20.5 & 33.8 & 43.3 & 43.3 \\
\hline 21 & 21.9 & 34.3 & 44.1 & 44.1 & 21.9 & 34.1 & 43.3 & 43.3 \\
\hline 22 & 44.1 & 44.1 & 44.1 & 44.1 & 43.3 & 43.3 & 43.3 & 43.3 \\
\hline 23 & 44.1 & 44.1 & 44.1 & 44.1 & 43.3 & 43.3 & 43.3 & 43.3 \\
\hline & 44.1 & 44.1 & 44.1 & 44.1 & 43.3 & 43.3 & 43.3 & 43.3 \\
\hline 25 & 44.1 & 44.1 & 44.1 & 44.1 & 43.3 & 43.3 & 43.3 & 43.3 \\
\hline
\end{tabular}
Grayscale Images

The received Image PSNR for varying channel conditions in terms of channel Noise $\mathrm{Eb} / \mathrm{No}$ is plotted in Figure 4 and Figure 6 for Lena and Babbon respectively. This is both for $2 \times 1$ and $2 \times 2$ MIMO systems. Their BER is calculated and plotted in Figure 5 and Figure 7 respectively. The PSNR improves with decreasing channel noise i.e., $\mathrm{E}_{b} / \mathrm{N}_{o}$ while the BER decreases over improving channel noise conditions. The high priority bits are not protected in EEP scheme hence they are prone to error. On the other hand, in UEP as we incorporate extra protection to high priority bit stream w.r.t. low priority bit stream, it leads to improved performance. 


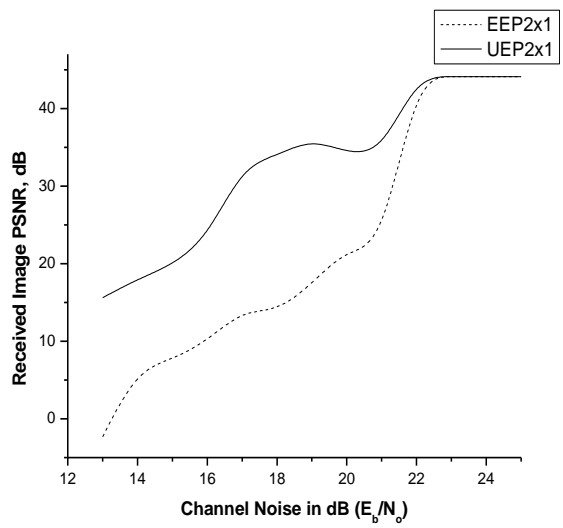

(a)

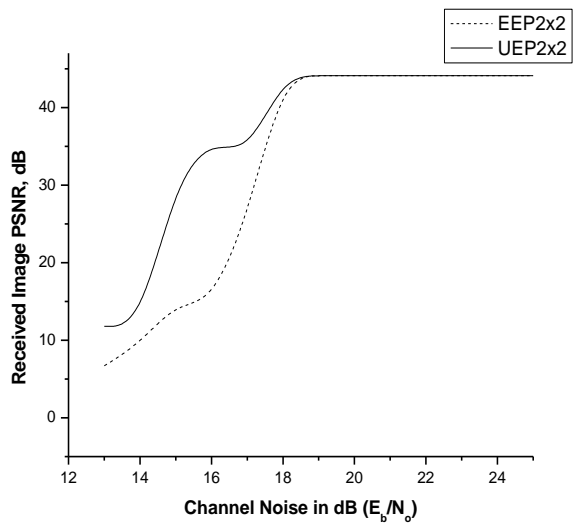

(b)

Figure 4. PSNR for Lena Image (a) with $2 \times 1\left(N_{T}=2, N_{R}=1\right)$ MIMO System and (b) with 2x2 ( $\left.\mathrm{N}_{\mathrm{T}}=2, \mathrm{~N}_{\mathrm{R}}=2\right)$ MIMO System

Figure 5. Bit Error Rate versus Channel $E_{b} / N_{o}$ for Lena Image

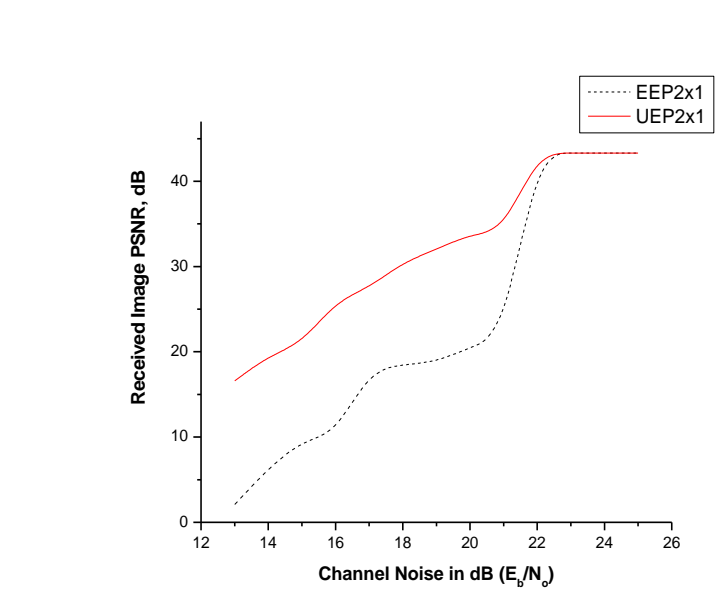

(a)

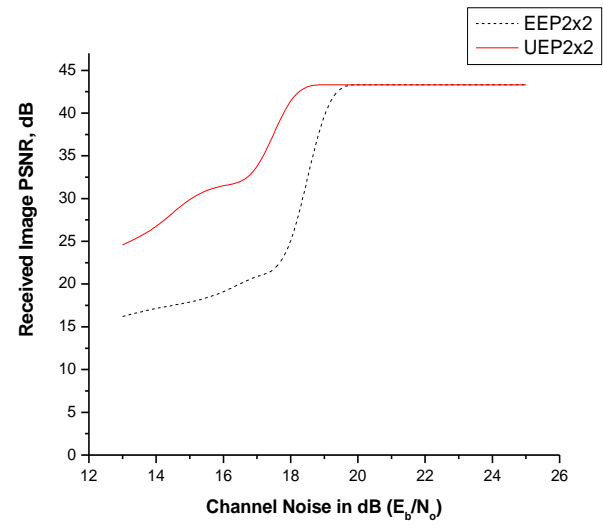

(b)

Figure 6. PSNR for Baboon Image (a) with $2 \times 1\left(N_{T}=2, N_{R}=1\right)$ MIMO System and (b) with $2 \times 2\left(N_{T}=2, N_{R}=2\right)$ MIMO System 


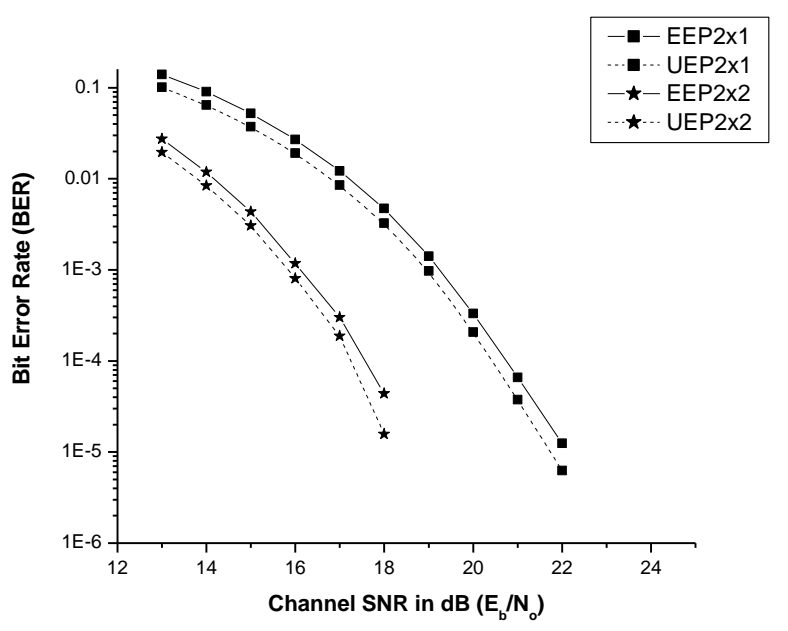

Figure 7. Bit Error Rate versus Channel $E_{b} / N_{o}$ for Baboon Image

A quantitative study to compare the performance of UEP w.r.t. EEP is presented in Table 2 and Table 3 for Lena and Baboon respectively at channel noise $16 \mathrm{~dB}$. The received images are also appended in Tables 2 and 3.

Table 2. Comparison of EEP and UEP Results for Lena Image

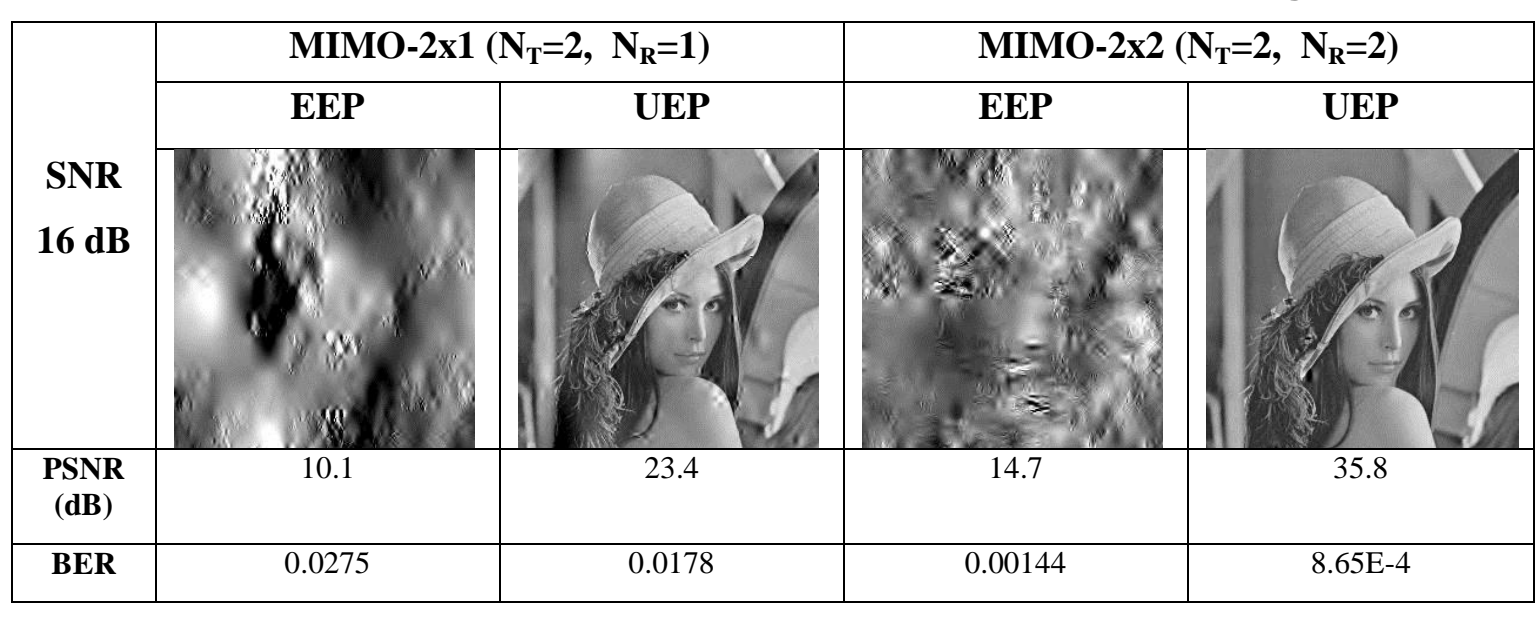

Table 3. Comparison of EEP and UEP Results for Baboon Image

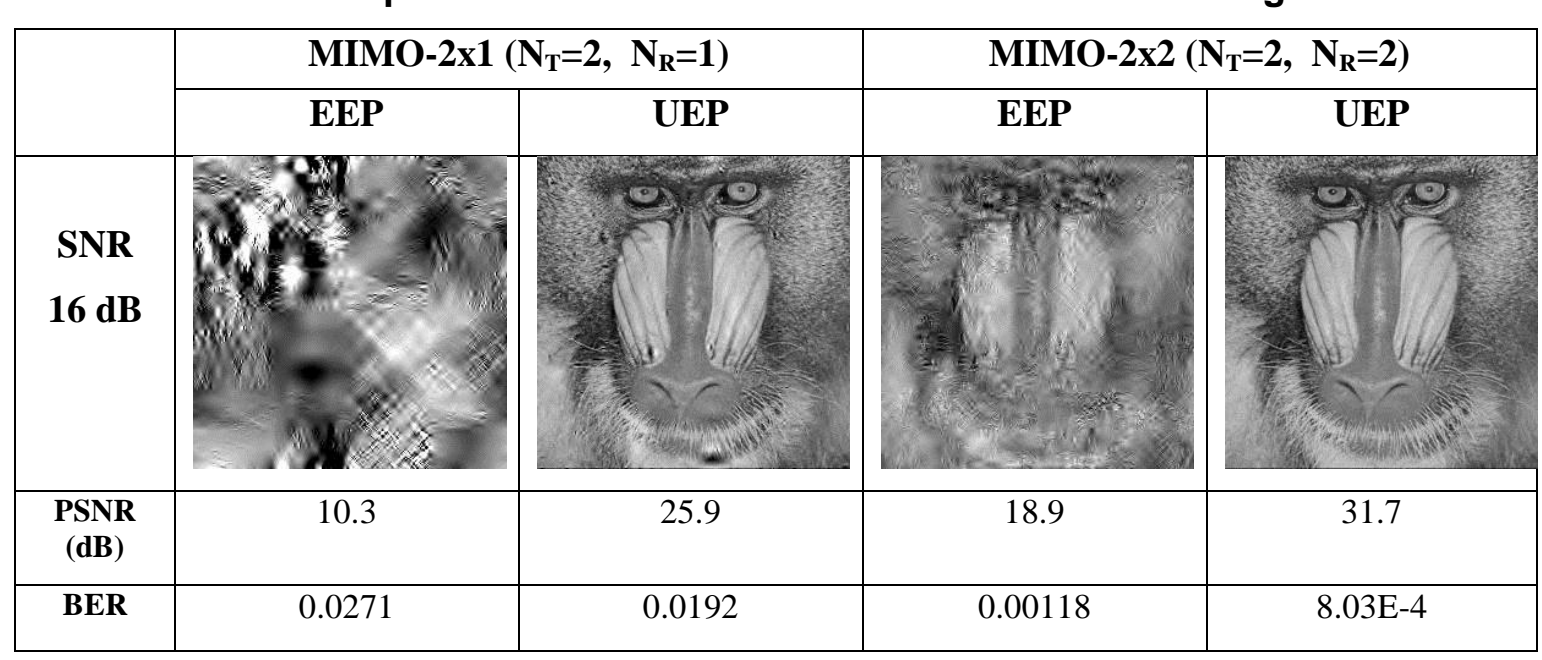


Note that, for both these images considerable improvement is observed in the visual quality of the received image in case of UEP. An improvement of $13 \mathrm{~dB}$ and $20 \mathrm{~dB}$ is observed respectively for $2 \mathrm{x} 1$ and 2x2 MIMO systems. This is presented in Table 2 . Similarly, data compiled in Table 3 shows an improvement of $15 \mathrm{~dB}$ and $13 \mathrm{~dB}$ for $2 \mathrm{x} 1$ \& $2 \times 2$ MIMO system respectively for Baboon image. The BER is found to decrease considerably both for Lena and Baboon as we switch from EEP to UEP. This clearly depicts considerable improvement in image quality obtained as a result of the present experimental setup.

Previous works done on UEP have focussed on joint channel source coding technique (JSCC) and mostly considered only AWGN channel in their work [12-14]. In the present work, we successfully use adaptive modulation scheme over SPIHT coded bitstream under more adverse Rayleigh fading channel conditions. The coded bit stream is transmitted over MIMO channel to achieve spatial and transmit diversity. The results show significant improvement for UEP scheme w.r.t. EEP scheme in received image quality. Results obtained using adaptive modulation technique are either on parer even better in some cases w.r.t. previous works done using JSCC. Fowdur et al., 12] use RCPC channel coding with different code-rates as well as prioritized retransmission of high priority bits over AWGN channel conditions. They report an average gain of $2 \mathrm{~dB}$ to $7 \mathrm{~dB}$ for different source coding rates of RCPC and by yarying retransmission frequency for high priority and low priority bit streams. The results obtained by âs in the present case clearly outperform their results. This is mainly attributed to enhanced protection given to the high priority bit stream which saves it from being corrupted in adverse channel conditions by using M-ary adaptive QAMmodulationscheme.

\section{Conclusion}

In this paper we propose a novel scheme for transmission of images over error prone fading channels using UEP scheme based upon significance of SPIHT source coded bit information and by varying modulation rates accordingly. The coded bit streams are transmitted over MIMO system to achieve better transmit and spatial diversity thereby further improving overalleceived image quality. The simulation results verify the proposed scheme to achieve UEP using adaptive modulation scheme. The present transmission scheme outperforms other similar scheme proposed by Fowdur et al., [12]. This is because it provided enlanced protection to the high priority bit stream as compared to loy priority bit stream which saves it from being corrupted under adverse Rayleigh fading channe conditions.

\section{References}

[1] Said and W. A. Pearlman, "A new, fast, and efficient image codec based on Set Partitioning in Hierarchical Trees," IEEE Trans. on Circuits \& Systems for Video Technology, vol. 6, no. 3, pp. 243 250 (1996) June.

[2] R.M. Pelz, "An unequal error protected kbit/s video transmission for DECT", Proceedings of the 44th EEE Vehicular Technology Conference 1994, (1994), pp. 1020-1024.

37. W. Modestino and D. G. Daut, "Combined source channel coding of images", IEEE Transactions on Communications, vol. 27, no. 11, (1979) November, pp. 1644- 1659.

[4] J. Hagenauer, "Rate-compatible punctured convolutional codes (RCPC codes) and their applications", IEEE Trans. Commun., vol. 36, (1988) April, pp. 389-400.

[5] A. Barbulescu and S. Pietrobon, "Rate compatible turbo codes," Electron. Lett., vol. 31, pp. 555-536, (1995) March.

[6] P. G. Sherwood and K. Zeger, "Progressive image coding on noisy channels", IEEE Signal Processing Letters, vol. 4, pp. 189-191, July 1997.

[7] H. Man, F. Kossentini and M. J. T. Smith, "A family of efficient and channel error resilient wavelet/subband image coders", IEEE Trans. Circuit \& Systems for Video Technology, vol. 9, pp. 95108, Feb. 1999.

[8] L. Cao, "On the Unequal Error Protection for Progressive Image Transmission", IEEE Transaction on Image Processing, vol. 16, no. 9, (2007) September, pp. 2384-2388. 
[9] M. A. Khan, A. A. Moinuddin, E. Khan and M. Ghanbari, "Error Analysis of SPIHT Based Image Coder", Proceedings of National Conference on Modern Trends in Electronics and Communication Systems (MTECS-05), AMU, India, (2005) March, pp. 104-110.

[10] J.-R. Ding and J.-F. Yang, "A Simplified SPIHT Algorithm", Journal of the Chinese Institute of Engineers, vol. 31, no. 4, (2008), pp 715-719.

[11] S. Alamouti, "A simple transmit diversity technique for wireless communications", IEEE Journal on selected areas in communications, vol. 16, no. 8, (1998), pp. 1451-1458.

[12] Fowdur, Indoonundon and Soyjaudah, "An Unequal Error Protection Scheme for SPIHT Image Transmission with Prioritized Retransmissions and De-noising", IEEE Conference AFRICON 2013, (2013) September, pp. 1-5.

[13] A. Khan and E. Khan, "Error Resilient Technique for SPIHT coded Color Images", IEEE Conference IMPACT-2009, (2009) March, pp. 237-240.

[14] Yap and Ngan, "Unequal Error Protection of images over rayleigh fading channels", Fifth International IEEE Symposium on Signal Processing and its Applications, IEEE Conference ISSPA '99, vol. 1, pp. 19-22, (1999) August.

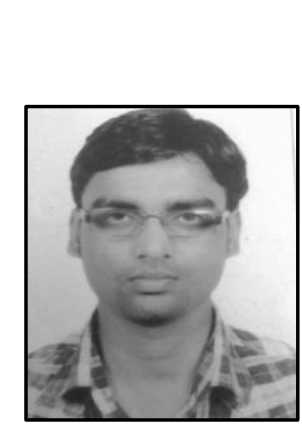

\section{Authors}

Rohit Kumar Murarka, received his B.Sc with Horors and M.Sc in Electronics from University of Delhi in year 2008 and 2010 respectively. He is currently pursuing M.Tech in Electronics and Communication Engineering from Gure Cobind Singh Indraprastha University, New Delhi. His research interests Include Image and Signal processing, Wireless Communication

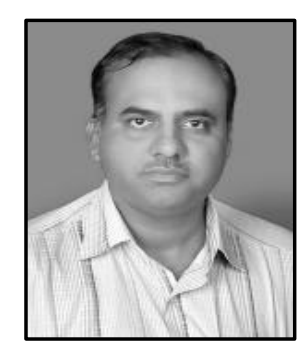

Anurag Mishrac received this Ph.D in Electronics from University of Delhi in 1995 He is currently working as Associate Professor in Deendayal Upadhyay College, University of Delhi. His research interests include informat)on security, digital watermarking of multimedia content and intelligent systems such as Artificial Neural Networks, Fuzzy Systems, Support Vector Machines and Extreme Learning Machines and evolutionary techniques such as Firefly algorithm He has developed novel image watermarking algorithms using hybrid systems such as Neuro - Fuzzy and GA - BPN systems. His new pnterest areas are Cyber Security and its applications.

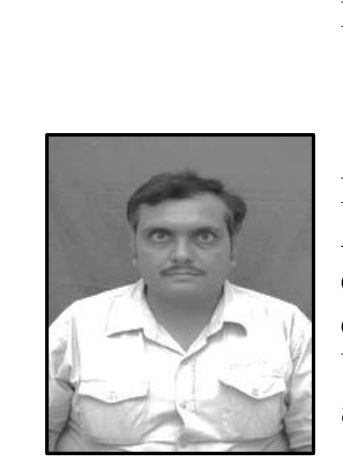

QKanchan Sharma, received his B.Tech and M.Tech degrees in Electronics and Communication engineering from University of Allahabad, Allahabad, India in 2000 and 2002 respectively. He is currently assistant professor with the department of electronics and communication engineering, Indira Gandhi Delhi Technical University for Women, New Delhi. His research interest is wireless and optical communication, image and signal processing. 
International Journal of Hybrid Information Technology

Vol.7, No.5 (2014)

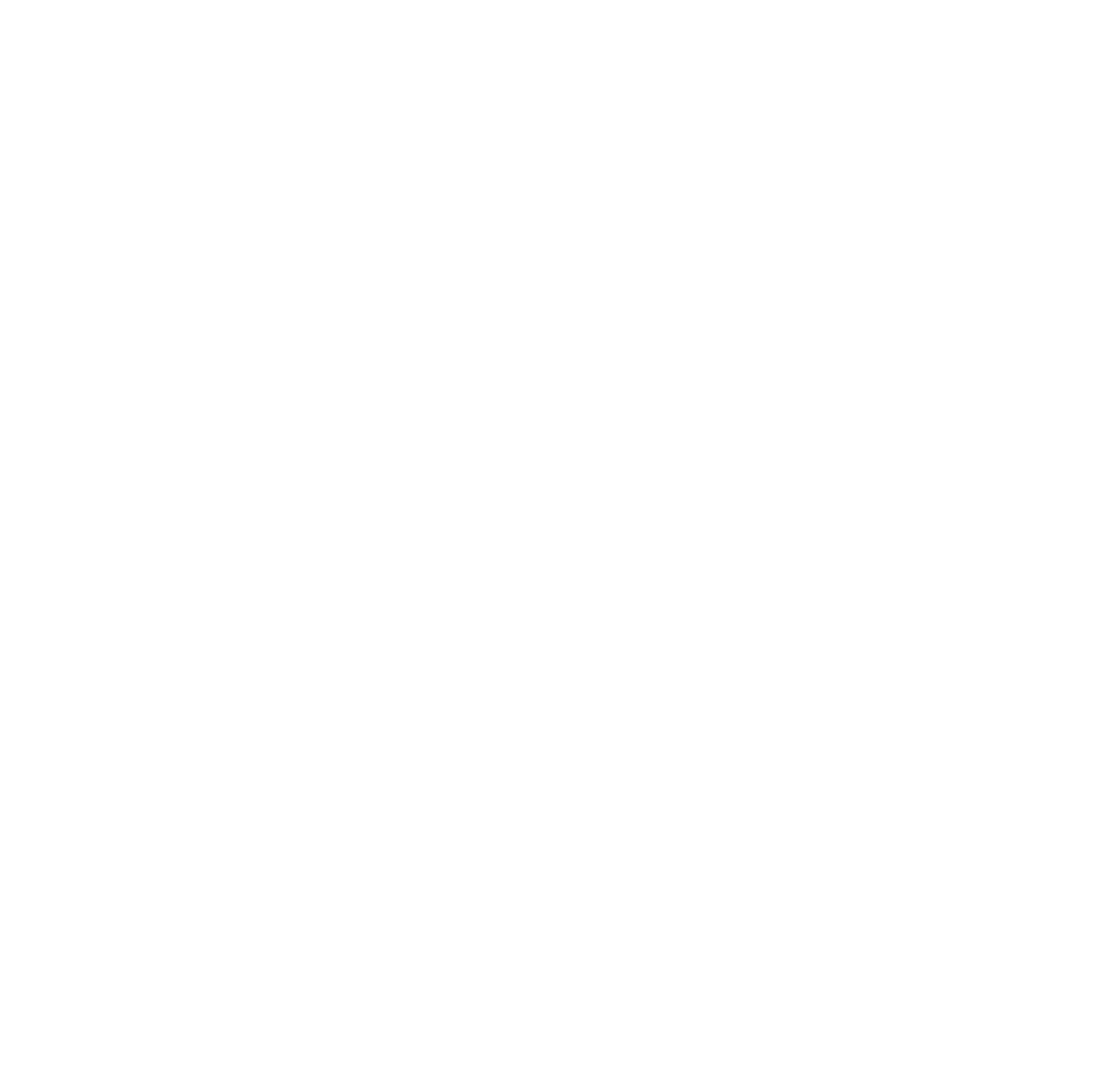

